\title{
Deviation From Delivery Of Radiation Therapy To Childhood Cancer Patients: A Retrospective Audit
}

\author{
Rashi Agrawal ${ }^{1}$, Vineeta Goel ${ }^{2}$, Anil Anand ${ }^{3}$, and Ramandeep Arora ${ }^{4}$ \\ ${ }^{1}$ Max Super Speciality Hospital Vaishali \\ ${ }^{2}$ Max Super Speciality Hospital Shalimar Bagh \\ ${ }^{3}$ Max Super Speciality Hospital Saket \\ ${ }^{4}$ Max Super-Speciality Hospital
}

April 12, 2021

\begin{abstract}
Background The delivery of Radiation therapy (RT) needs a level of coordination and planning which can be challenging in resource-limited settings. In this retrospective study we describe the profile of children undergoing radiation and analyze deviation from accepted norms. Procedure Data on all children ( $<18$ years age) with cancer who completed RT from Jan 2009 to Dec 2019 were retrieved. Diagnostic groups with more than 5 patients, were included in the analysis for deviations in RT (Time to start (TTS); total dose delivered (TDD) in Grays; and time to complete (TTC)). We investigated reasons for deviation. Results 207 received RT as front-line treatment (68\% Indian, 59\% male and 32\% received treatment preceding RT outside). Most common diagnosis were brain tumors (44\%), lymphoma (13\%). leukemia and soft tissue sarcoma (10\% each). $23.6 \%$ had deviation in TTS, $6.0 \%$ in TDD and $24.7 \%$ in TTC while $43.4 \%$ had at least one deviation in any of these three parameters. Deviation in TTS varied significantly by location of preceding treatment and by cancer (greatest deviation in sarcomas) with issues around access to healthcare the most common reason. Deviation in TTC varied significantly by cancer (greatest deviation in sarcomas and medulloblastoma) with myelotoxicity the most common reason. Conclusions Our study adds to the limited literature on RT quality for children with cancer in LMIC. Certain cancers (sarcomas and medulloblastoma) and patient groups (preceding treatment outside our institute) had the maximum deviation. Barriers to accessing care and myelotoxicity were the two main reasons for this deviation.
\end{abstract}

\section{TITLE PAGE}

Title

Deviation From Delivery Of Radiation Therapy To Childhood Cancer Patients: A Retrospective Audit

Authors

Rashi Agrawal, ${ }^{1}$ Vineeta Goel, ${ }^{2}$ Anil Anand, ${ }^{3}$ Ramandeep Singh Arora ${ }^{3}$

Affiliations

1. Max Institute of Cancer Care, Max Super Speciality Hospital, Vaishali, Ghaziabad

2. Max Institute of Cancer Care, Max Super Speciality Hospital, Shalimar Bagh, New Delhi

3. Max Institute of Cancer Care, Max Super Speciality Hospital, Saket, New Delhi

Corresponding Author

Dr Rashi Agrawal, Radiation Oncology, Max Super Speciality Hospital, Vaishali, Ghaziabad, Uttar Pradesh, India 201012, Tel +91 9891483550, Email drrashi.ag@gmail.com 
Word Count Abstract 332

Word Count Main Text 1746

Number of Tables 4

Number of Figures 0

Number of Supporting Information files 0

Short running title

Radiation Delivery Deviations

Keywords

Childhood Cancer; Radiotherapy; Clinical Audit; Delivery of Health Care; India

All the authors have no conflict of interest.

Abbreviations

\begin{tabular}{ll}
\hline RT & Radiotherapy \\
\hline TTS & Time to start \\
TDD & Total dose delivered \\
TTC & Time to complete \\
LMIC & Low and middle-income countries \\
ICCC & International Childhood Cancer Classification \\
\hline
\end{tabular}

\section{Background}

The delivery of Radiation therapy (RT) needs a level of coordination and planning which can be challenging in resource-limited settings. In this retrospective study we describe the profile of children undergoing radiation and analyze deviation from accepted norms.

\section{Procedure}

Data on all children ( $<18$ years age) with cancer who completed RT from Jan 2009 to Dec 2019 were retrieved. Diagnostic groups with more than 5 patients, were included in the analysis for deviations in RT (Time to start (TTS); total dose delivered (TDD) in Grays; and time to complete (TTC)). We investigated reasons for deviation.

\section{Results}

207 received RT as front-line treatment (68\% Indian, $59 \%$ male and $32 \%$ received treatment preceding RT outside). Most common diagnosis were brain tumors (44\%), lymphoma (13\%). leukemia and soft tissue sarcoma (10\% each). $23.6 \%$ had deviation in TTS, $6.0 \%$ in TDD and $24.7 \%$ in TTC while $43.4 \%$ had at least one deviation in any of these three parameters. Deviation in TTS varied significantly by location of preceding treatment and by cancer (greatest deviation in sarcomas) with issues around access to healthcare the most common reason. Deviation in TTC varied significantly by cancer (greatest deviation in sarcomas and medulloblastoma) with myelotoxicity the most common reason.

\section{Conclusions}

Our study adds to the limited literature on RT quality for children with cancer in LMIC. Certain cancers (sarcomas and medulloblastoma) and patient groups (preceding treatment outside our institute) had the maximum deviation. Barriers to accessing care and myelotoxicity were the two main reasons for this deviation. Introduction 
The role and need for radiation therapy (RT) of childhood cancer treatment is well established.(1) Despite declining trends of use over time,(2) it remains a critical part of multimodality treatment for several childhood cancers.(1) Modern treatment protocols are complex and multidisciplinary and often prescribe in detail the indication, the timing, the field and the dose of RT with additional guidance on managing toxicity.

The delivery of RT thus needs a level of coordination and planning which can be challenging in resourcelimited settings.(3) This is because globally access to RT is not uniform and there are deficiencies in availability of RT machines, trained staff, as well as aspects of quality like input of interdisciplinary RT teams, treatment planning protocols, and quality assurance. $(3,4)$ This is an under-researched area in low and middleincome countries (LMIC) including India where most of the childhood cancer cases occur.

In this retrospective study we audit the profile of children undergoing RT at our centre and analyze deviation from accepted norms during their treatment. This information will provide us guidance on how to further improve the delivery of our RT services.

\section{Methods}

This audit was conducted at three units of Max Institute of Cancer Care (MICC) Saket, Patparganj and Vaishali which are all healthcare establishments in the private sector located in Delhi National Capital Region. RT in these units was delivered on the Linac accelerator by different techniques. Thermoplastic mould and Vac-loc were used as immobilization device for simulation and treatment for each patient. Patients received five fractions of external beam radiotherapy each week. CT based planning for treatment was done for every patient irrespective of the technique. Anaesthesia for immobilisation and radiation delivery for young children was used as per requirement.

Children with cancer ( $<18$ years of age) treated with RT from 1st January 2009 to $31^{\text {st }}$ December 2019 in these units were identified. Those who received RT in a relapsed/refractory setting were excluded as were those where the RT was given to the metastatic site only (e.g. lung metastasis). Relevant demographic, clinical and RT data was retrieved from RT records, clinical notes and the pediatric hospital based case registry. Patients were categorized as per International Childhood Cancer Classification (ICCC).(5)

From this cohort, we further selected those ICCC categories and sub-categories where there were at least 5 patients to analyze deviation from accepted norms during their treatment. We looked at three specific parameters in these patients: Time to start (TTS) RT calculated as the time interval between date of diagnosis and start of RT; total dose delivered (TDD) in Grays; and time to complete (TTC) RT which is the interval between the first fraction and the last fraction of the scheduled RT.

For the purpose of this audit, we had to create a standard. Two of the authors (RA and RSA) examined recommendations of contemporary national and international protocols as well as a standard textbook of radiation oncology.(6) From this standards on TTS and TDD were created. These were then reviewed by the other two authors (VG and AKA) and finalised (Table 1). Deviation was defined as

- For TTS - If greater than standards in Table 1 unless preceding surgery or chemotherapy was delayed or treatment toxicity occurred which then impacted on TTS.

- For TDD - If dose of radiation was lesser or greater than standards in Table 1.

- For TTC - If the duration of radiation was $5 \%$ more than the total number of fractions prescribed with allowance for weekends.

We further examined association deviation with demographic (age, gender, nationality), disease (diagnosis) ad treatment (whether preceding treatment was done at MICC or not) variables. Excel sheet was used for data collection and chi square test was applied to see association between variables. $\mathrm{P}$ value less than or equal to 0.05 was considered significant. We also examined any reasons for deviation which could be identified through searching of RT records and clinical notes. The analysis was descriptive and results were tabulated for display.

As this was a retrospective audit, patient consent was not required. 


\section{Results}

A total of 275 children with cancer ( $<18$ years of age) received RT from 1st January 2009 to $31^{\text {st }}$ December 2019 in the three MICC units. Of these 68 (24.7\%) were excluded (RT was given for refractory/relapse disease $(n=64)$, for palliation of metastatic site $(n=3)$ and for lung metastasis $(n=1))$ and 207 were included (59\% male, mean age 9.3 years, $68 \%$ from India, $67 \%$ received preceding treatment at MICC). The most common ICCC categories were CNS tumours (44\%), lymphomas (13\%), leukemias and soft tissue sarcomas (10\% each), bone sarcomas (8\%) and all others (15\%).

Of the 207 included patients, 182 were examined for deviation of TTS, TDD and TTC as they belonged to the 13 diagnoses in which there were more than five patients (table 2 and table 3). The mean TTS, TTD and TTC are displayed in table 2. 43 children (23.6\%) had deviation in TTS, 11 (6.0\%) in TDD and $45(24.7 \%)$ in TTC while 79 children $(43.4 \%)$ had at least one deviation in any of these three parameters. Deviation from these and their association with age, gender, nationality, type of cancer and preceding treatment are displayed in Table 3. Type of cancer was significantly associated with deviation in all three parameters. TTS deviations were greatest in Ewing sarcoma and nonrhabdomyosarcoma soft tissue sarcoma, and renal tumours; TDD deviations were greatest in brain stem gliomas; and TTC deviations were greatest in nonrhabdomyosarcoma soft tissue sarcoma, medulloblastoma and primitive neuroectodermal tumour, and Ewing sarcoma. In addition location of preceding treatment and nationality were also significantly associated with deviation of TTS.

Table 4 lists the reasons attributed to these deviations. TTS was mostly impacted by issues around accessing care like preceding treatment outside our centres, logistical challenges of finance or approval for treatment, and travelling home or for second opinion. No specific reason was identified for TDD deviations. Myelotoxicity was the most common reason for deviations in TTC but we could not establish the reason in a third of the patients.

\section{Discussion}

Quality assurance is integral to modern day delivery of RT and focusses on metrics related to processes, logistics and timing. RT protocol compliance is one aspect of quality assurance and adult and paediatric studies have confirmed that RT protocol deviation leads to increased treatment failure $(7,8)$. There is no accepted universal definition of deviations and this varies by cancer and protocol but would generally include one or more of the following; dose, volume, field, timing of start and overall treatment time $(7,8)$.

In our audit of children with cancer who received RT at our institute we found that approximately one in two children had a deviation in one parameter with one in four children having deviation in TTS and TTC but only one in 17 having deviation in TDD. A recent study from the Dominican Republic with a relatively smaller sample size (19 children with Wilms tumour, rhabdomyosarcoma, retinoblastoma and nasopharyngeal carcinoma) is the only other study from LMIC which has previously studied this and found that $95 \%$ of children had at least one deviation (they also examined an additional parameter of field) with greatest deviation in TTC $79 \%$ followed by TTS $53 \%$ and finally TDD $42 \%$.(9)

These findings are concerning because the published literature provides evidence to the importance of these parameters. Delay in starting RT impacts outcome of children with Ewing sarcoma(10-12), Wilms tumour(13) and medulloblastoma(14) and excess time taken to complete RT has an adverse impact on children with medulloblastoma.(15) Similarly dose deviations have had an adverse impact in children with rhabdomyosarcoma(16), medulloblastoma(17) and Ewing sarcoma.(18) A limitation of our study is that we did not have follow-up for each patient to determine if deviations had an impact on outcomes but it would not be incorrect to assume some detrimental impact.

Factors associated with deviations may be demographic, disease or treatment related. We found the greatest deviations in TTS in sarcomas in comparison to leukemia, lymphoma and CNS tumours. This may be due to involvement of both, surgery and chemotherapy as modalities before radiation and toxicities related to these modalities. In carcinoma nasopharynx and brainstem glioma, in which radiation was primary mode of 
treatment, treatment was started within days of diagnosis.

Additionally, healthcare related factors, which may be specific to our institution as well as the wider context of healthcare delivery in the country, may impact these deviations. $31 \%$ of the children who received RT had their preceding treatment (surgery and/or chemotherapy) outside our institute and their deviation in TTS was double of those who had preceding treatment at our institute. This highlights the lack of access to RT and the need to move to different locations, compromising delivery of quality care. Once RT commenced at our institute, preceding treatment ceased to be a significant factor in deviation of TTC as our infrastructure is geared to deliver uninterrupted treatment if the child remains well.

Similarly deviations in TDD were only around $6 \%$ and less likely to be impacted by any of the factors mentioned above. Although most of our patients are not on prospective clinical studies, we do use national and international recommendations and protocols to deliver the correct doses. Our data does show that there was TDD deviation in brainstem gliomas which received a mean dose of 52.6 Gray against a recommended dose of 54 to 60 Gray. We are now putting in guidelines to correct this.

We did see significant association of cancer type with TTC with the high degree of deviation seen in medulloblastoma where craniospinal irradiation (CSI) would lead to myelotoxicity. This challenge has also been highlighted in another publication from India where $73 \%$ of patients had interruptions in CSI.(19) In contrast experience from USA suggests that interruptions even in this group of patients can be as low as $2.5 \%$.(20) We plan to review our practice of CSI including thresholds for interrupting RT and use of growth factors to address this.

In conclusion, our study adds to the limited literature on RT quality for children with cancer in LMIC. It highlights that one in four children have deviation in TTS and TTC RT and provides us areas to improve within our practice. Our work is limited by the fact that we did not examine the parameter of volume or field. Also our study is retrospective and dependent on the quality of record keeping and so we cannot comment on the individual circumstances which may have led to the deviation (e.g. site and stage of tumour, treatment intensity, etc.).

\section{Acknowledgements}

We would like to acknowledge Prof Abhaya Indrayan for his help with the statistical analysis and Dr Himanshu, Dr Sahil, Dr Naman Utreja for assisting in some of the data collection.

\section{Conflict of Interest statement}

All the authors have no conflict of interest to declare

\section{References}

1. Gibbs IC, Tuamokumo N, Yock TI. Role of radiation therapy in pediatric cancer. Hematol Oncol Clin North Am. 2006 Apr;20(2):455-70.

2. Jairam V, Roberts KB, Yu JB. Historical trends in the use of radiation therapy for pediatric cancers: 1973-2008. Int J Radiat Oncol Biol Phys. 2013 Mar 1;85(3):e151-5

3. Parkes J, Hess C, Burger H, Anacak Y, Ahern V, Howard SC, Elhassan M, Ahmed S, Ghalibafian M, Abbasi AN, Qureshi BM, Zaghloul M, Zubizarreta E, Bey P, Davidson A, Bouffet E, Esiashvili N. Recommendations for the treatment of children with radiotherapy in low- and middle-income countries (LMIC): A position paper from the Pediatric Radiation Oncology Society (PROS-LMIC) and Pediatric Oncology in Developing Countries (PODC) working groups of the International Society of Pediatric Oncology (SIOP). Pediatr Blood Cancer. 2017 Dec;64 Suppl 5. doi: 10.1002/pbc.26903. PMID: 29297617.

4. Anacak Y, Zubizarreta E, Zaghloul M, Laskar S, Alert J, Gondhowiardjo S, Giselvania A, CorreaVillar R, Pedrosa F, Dorj B, Kamer S, Howard SC, Quintana Y, Ribeiro RC, Rosenblatt E, Hopkins K. The Practice of Paediatric Radiation Oncology in Low- and Middle-income Countries: Outcomes 
of an International Atomic Energy Agency Study. Clin Oncol (R Coll Radiol). 2020 Nov 26:S09366555(20)30418-0.

5. Steliarova-Foucher E, Stiller C, Lacour B, Kaatsch P. International Classification of Childhood Cancer, third edition. Cancer. 2005 Apr 1;103(7):1457-67.

6. Constine LS, Tarbell NJ, Halperin EC. Pediatric radiation oncology. Lippincott Williams \& Wilkins; $2016 \mathrm{Jul} 5$.

7. Fairchild A, Straube W, Laurie F, Followill D. Does quality of radiation therapy predict outcomes of multicenter cooperative group trials? A literature review. Int J Radiat Oncol Biol Phys. 2013 Oct 1;87(2):246-60.

8. Ohri N, Shen X, Dicker AP, Doyle LA, Harrison AS, Showalter TN. Radiotherapy protocol deviations and clinical outcomes: a meta-analysis of cooperative group clinical trials. J Natl Cancer Inst. 2013 Mar 20;105(6):387-93.

9. Power-Hays A, Friedrich P, Fernandez G, Cruz NA, Marcus K, Rodriguez-Galindo C, Collado L. Delivery of radiation therapy in resource-limited settings: A pilot quality assessment study. Pediatr Blood Cancer. 2017 Aug;64(8):10.1002/pbc.26480.

10. Totadri S, Bansal D, Rao KLN, Jain R, Saxena AK, Kapoor R, Samujh R, Trehan A. Challenges in the management of localized Ewing sarcoma in a developing country. Pediatr Hematol Oncol. 2020 Oct;37(7):610-619

11. Parambil BC, Vora T, Sankaran H, Prasad M, Bakshi A, Puri A, Gulia A, Qureshi S, Laskar S, Khanna N, Shah S, Ramadwar M, Kembhavi S, Chinnaswamy G, Banavali S. Outcomes with nondose-dense chemotherapy for Ewing sarcoma: A practical approach for the developing world. Pediatr Blood Cancer. 2020 Nov;67(11):e28604.

12. Lin TA, Ludmir EB, Liao KP, McAleer MF, Grosshans DR, McGovern SL, Bishop AJ, Woodhouse KD, Paulino AC, Yeboa DN. Timing of Local Therapy Affects Survival in Ewing Sarcoma. Int J Radiat Oncol Biol Phys. 2019 May 1;104(1):127-136.

13. Stokes CL, Stokes WA, Kalapurakal JA, Paulino AC, Cost NG, Cost CR, Garrington TP, Greffe BS, Roach JP, Bruny JL, Liu AK. Timing of Radiation Therapy in Pediatric Wilms Tumor: A Report From the National Cancer Database. Int J Radiat Oncol Biol Phys. 2018 Jun 1;101(2):453-461.

14. Kortmann RD, Kühl J, Timmermann B, Mittler U, Urban C, Budach V, Richter E, Willich N, Flentje M, Berthold F, Slavc I, Wolff J, Meisner C, Wiestler O, Sörensen N, Warmuth-Metz M, Bamberg M. Postoperative neoadjuvant chemotherapy before radiotherapy as compared to immediate radiotherapy followed by maintenance chemotherapy in the treatment of medulloblastoma in childhood: results of the German prospective randomized trial HIT '91. Int J Radiat Oncol Biol Phys. 2000 Jan 15;46(2):269-79.

15. Taylor RE, Bailey CC, Robinson K, Weston CL, Ellison D, Ironside J, Lucraft H, Gilbertson R, Tait DM, Walker DA, Pizer BL, Imeson J, Lashford LS; International Society of Paediatric Oncology; United Kingdom Children's Cancer Study Group. Results of a randomized study of preradiation chemotherapy versus radiotherapy alone for nonmetastatic medulloblastoma: The International Society of Paediatric Oncology/United Kingdom Children's Cancer Study Group PNET-3 Study. J Clin Oncol. 2003 Apr 15;21(8):1581-91.

16. Million L, Anderson J, Breneman J, Hawkins DS, Laurie F, Michalski J, Rodeberg D, Wharam M, Wolden S, Donaldson SS; Soft Tissue Sarcoma Committee of the Children's Oncology Group. Influence of noncompliance with radiation therapy protocol guidelines and operative bed recurrences for children with rhabdomyosarcoma and microscopic residual disease: a report from the Children's Oncology Group. Int J Radiat Oncol Biol Phys. 2011 Jun 1;80(2):333-8. 
17. Carrie C, Hoffstetter S, Gomez F, Moncho V, Doz F, Alapetite C, Murraciole X, Maire JP, Benhassel M, Chapet S, Quetin P, Kolodie H, Lagrange JL, Cuillere JC, Habrand JL. Impact of targeting deviations on outcome in medulloblastoma: study of the French Society of Pediatric Oncology (SFOP). Int J Radiat Oncol Biol Phys. 1999 Sep 1;45(2):435-9.

18. Donaldson SS, Torrey M, Link MP, Glicksman A, Gilula L, Laurie F, Manning J, Neff J, Reinus W, Thompson E, Shuster JJ. A multidisciplinary study investigating radiotherapy in Ewing's sarcoma: end results of POG \#8346. Pediatric Oncology Group. Int J Radiat Oncol Biol Phys. 1998 Aug 1;42(1):12535.

19. Kumar N, Miriyala R, Thakur P, Madan R, Salunke P, Yadav B, Gupta A. Impact of acute hematological toxicity on treatment interruptions during cranio-spinal irradiation in medulloblastoma: a tertiary care institute experience. J Neurooncol. 2017 Sep;134(2):309-315.

20. Chang EL, Allen P, Wu C, Ater J, Kuttesch J, Maor MH. Acute toxicity and treatment interruption related to electron and photon craniospinal irradiation in pediatric patients treated at the University of Texas M. D. Anderson Cancer Center. Int J Radiat Oncol Biol Phys. 2002 Mar 15;52(4):1008-16.

21.

\section{Legends}

Table 1 Radiation Reference Doses and Timing for Childhood Cancers

Table 2 Mean time to start radiation, dose of radiation and time to complete radiation by type of cancer

Table 3 Association of deviation of mean time to start radiation, dose of radiation and time to complete radiation with different variables

Table 4 Reasons attributed to deviation of time to start radiation, dose of radiation and time to complete radiation

\section{Hosted file}

Table 1 Radiation Reference Doses and Timing for Childhood Cancers.pdf available at https://authorea.com/users/407426/articles/517741-deviation-from-delivery-of-radiationtherapy-to-childhood-cancer-patients-a-retrospective-audit

\section{Hosted file}

Table 2 Mean time to start radiation.pdf available at https://authorea.com/users/407426/ articles/517741-deviation-from-delivery-of-radiation-therapy-to-childhood-cancerpatients-a-retrospective-audit

\section{Hosted file}

Table 3 Association of deviation of mean time to start radiation.pdf available at https: //authorea.com/users/407426/articles/517741-deviation-from-delivery-of-radiationtherapy-to-childhood-cancer-patients-a-retrospective-audit

\section{Hosted file}

Table 4 Reasons attributed to deviation of time to start radiation.pdf available at https://authorea. com/users/407426/articles/517741-deviation-from-delivery-of-radiationtherapy-to-childhood-cancer-patients-a-retrospective-audit 